\title{
Analysis of Efficiency upon Enhancing the Fuel Combustion Completeness in the GTU Burners Using Fuel Gas Heating up
}

\author{
Pekov A.P., Bachev N.L., Shilova A.A., Matyunin O.O., Betinskaya O.A. \\ Perm National Research Polytechnic University \\ Perm, Russian Federation
}

\begin{abstract}
One main characteristic of the gas turbine unit (GTU) burner is its fuel combustion completeness, which affects directly the efficiency of the power plant along with $\mathrm{CO}$ and unburnt hydrocarbons $\mathrm{C}_{\mathrm{n}} \mathrm{H}_{\mathrm{m}}$ emissions. The aim of this work was the research on the application of the fuel heating-up as an alternative method for increasing the fuel combustion completeness and controlling the emission of harmful agents. This goal is achieved by obtaining experimental data on the emissions of $\mathrm{CO}$ and $\mathrm{NO}_{\mathrm{x}}$ at different temperatures of the fuel gas supply to the combustion chamber. The most significant result of the work is the experimentally confirmed possibility of increasing the combustion efficiency (decreasing $\mathrm{CO}$ ) by heating the fuel gas while maintaining constant gas-dynamic characteristics of the chamber. The significance of the results obtained consists in the experimental confirmation of the combustion quality control only by heating the fuel gas without changing the operating and design characteristics of the combustion chamber. The fuel combustion low completeness can cause the burner unstable operation in the form of the unsteady pre-blowout burning combined with the pressure oscillations in the burner. At present, methods for ensuring the increase in stability and completeness of the fuel combustion are related to the air rate and temperature changes at the inlet. However, the use of these methods can be unwanted because of their causing the decrease in the coefficient of efficiency and in the resource of the 'hot part' of the gas-turbine facility.

Keywords: fuel gas heating-up, fuel combustion completeness, harmful agents emission, experimental facility, torch unit.
\end{abstract}

DOI: https://doi.org/10.52254/1857-0070.2021.4-52.09

UDC: $\mathbf{5 3 6 . 4 6 4}$

\section{Analiza eficienței creșterii plenitudinii arderii combustibilului în camerele de ardere ale instalațiilor turbinelor cu gaz prin preîncălzirea gazelor combustibile \\ Pekov A.P., Bacev N.L., Șilova A.A., Matiunin O.O., Betinskaia O.A. \\ Universitatea națională de cercetare politehnică din Permi \\ Permi, Federatia Rusă}

Rezumat. Una dintre principalele caracteristici ale camerei de ardere ale instalației turbinei cu gaz este plenitudinea arderii combustibilului, de care în mod direct depinde eficiența instalației energetice și emisiile de monoxid de carbon $\mathrm{CO}$ și de hidrocarburi nearse $\mathrm{C}_{\mathrm{n}} \mathrm{H}_{\mathrm{m}}$. Scopul lucrării este cercetarea utilizării încălzirii combustibilului ca mijloc alternativ de creștere a plenitudinii arderii combustibilului și de control al emisiilor de substanțe nocive. Obiectivul este atins prin obținerea de date experimentale privind emisiile de $\mathrm{CO}$ și $\mathrm{NO}_{\mathrm{x}}$ la diferite temperaturi de alimentare a camerei de ardere cu gaz combustibil. Cel mai important rezultat al lucrării este posibilitatea dovedită experimental de a crește plenitudinea combustiei (reducerea emisiilor de $\mathrm{CO}$ ) prin încălzirea gazului combustibil, menținând constante caracteristicile gazodinamice și geometrice ale camerei de ardere. Importanța rezultatelor obținute constă în confirmarea experimentală a posibilității de a controla calitatea combustiei doar prin încălzirea gazului combustibil fără a schimba regimul și caracteristicile de proiectare ale camerei de ardere. Plenitudinea joasă de ardere a combustibilului poate cauza o funcționare instabilă a camerei de ardere sub forma unei combustii instabile înainte de ardere, însoțită de fluctuații de presiune în camera de ardere. O eficiență ridicată a combustiei este deosebit de importantă în camerele de ardere cu emisii reduse. La regimuri înalte de funcționare, pentru a menține cea mai mică emisie de $\mathrm{NO}_{x}$ este necesară menținerea unei temperaturi minime a flăcării stabile. Însă aplicarea metodelor indicate duce la consecințe nedorite, exprimate în reducerea randamentului și a resursei ,,părții fierbinți” a instalaţiei turbinei pe gaz.

Cuvinte-cheie: preîncălzirea gazelor combustibile, plenitudinea arderii combustibilului, emisii de substanțe nocive, instalație pilot, dispozitiv de ardere.

(C) Пеков А.П., Бачев Н.Л.,

Шилова А.А., Матюнин О.О.,

Бетинская О.A. 


\section{Анализ эффективности повышения полноты сгорания топлива в камерах сгорания ГТУ путем подогрева топливного газа}

Пеков А.П., Бачев Н.Л., Шилова А.А., Матюнин О.О., Бетинская О.А.

Пермский национальный исследовательский политехнический университет Пермь, Российская Федерация

Аннотация. Одной из основных характеристик камеры сгорания газотурбинной установки является полнота сгорания топлива, от которой напрямую зависит экономичность энергоустановки и эмиссия моноокиси углерода $\mathrm{CO}$ и несгоревших углеводородов $\mathrm{C}_{\mathrm{n}} \mathrm{H}_{\mathrm{m}}$. Целью данной работы являются исследования по применению подогрева топлива в качестве альтернативного способа повышения полноты сгорания топлива и управления эмиссией вредных веществ. Поставленная цель достигается получением экспериментальных данных по эмиссии $\mathrm{CO}$ и $\mathrm{NO}_{x}$ при разных температурах подачи топливного газа в камеру сгорания. Наиболее существенным результатом работы является экспериментально подтвержденная возможность повышения полноты сгорания (уменьшения эмиссии СО) путем подогрева топливного газа при сохранении постоянных газодинамических и геометрических характеристик камеры сгорания. Значимость полученных результатов состоит в экспериментальном подтверждении возможности управления качеством горения только подогревом топливного газа без изменения режимных и конструктивных характеристик камеры сгорания. Низкая полнота сгорания топлива может быть причиной неустойчивой работы камеры сгорания в виде нестабильного предсрывного горения, сопровождающегося колебаниями давления в камере сгорания. Обеспечение высокой полноты сгорания топлива особенно важно в малоэмиссионных камерах сгорания. На высоких режимах работы для обеспечения минимально возможной эмиссии $\mathrm{NO}_{x}$ необходимо обеспечивать минимальную возможную температуру стабильного пламени. На сегодняшний день на практике широко применяются следующие способы повышения устойчивости и полноты сгорания топлива: локальное повышение концентрации топлива в факеле пламени путем сброса части воздуха в турбину помимо горелок, сброса части воздуха из камеры в атмосферу и путем отключения части горелок, а также их комбинации. Однако применение указанных способов приводит к нежелательным последствиям, выражающимся в снижении КПД и ресурса «горячей части» газотурбинной установки.

Ключевые слова: подогрев топливного газа, полнота сгорания топлива, эмиссия вредных веществ, экспериментальная установка, горелочное устройство.

\section{ВВЕДЕНИЕ}

Bce известные способы управления качеством и устойчивостью горения в камерах сгорания (КС) газотурбинных установок (ГТУ) основаны на снижении времени индукции топлива и повышении скорости его сгорания [1]. По способу физического воздействия на процесс горения эти способы одинаковы - они изменяют температуру горения либо за счет изменения температуры горюче-воздушной смеси (ГВС), либо за счет изменения коэффициента избытка воздуха [2]. Подогрев воздуха и топливного газа перед их подачей в КС поразному влияет на характеристики внутрикамерного процесса и режимные параметры ГТУ. Температура подогретого воздуха сильно влияет на температуру ГВС и энергоэффективность установки, а температура подогретого топливного газа сильно сдвигает нижний предел горения обедненных ГВС в сторону больших значений коэффициента избытка воздуха.

О влиянии температуры воздуха и топливного газа на газодинамические и эмиссионные характеристики горения опубликованы в основном экспериментальные данные. В работе
[3] приведены результаты экспериментальных исследований по влиянию температуры воздуха на характеристики горения в КС микрогазотурбинной установки. Результаты показывают, что с увеличением температуры воздуха эффективность сгорания увеличивается, выбросы $\mathrm{CO}$ и $\mathrm{C}_{\mathrm{n}} \mathrm{H}_{\mathrm{m}}$ значительно снижаются, а выбросы $\mathrm{NO}_{x}$ значительно увеличиваются. В исследовании [4] экспериментально изучалось влияние подогрева воздуха и состава топлива на характеристики горения в КС модельной ГТУ. Температура воздуха изменялась в диапазоне от 383 К до 483 К при фиксированном коэффициенте избытка воздуха, равного 1.2. Выявлено, что КС может перейти в термоакустическое нестабильное состояние, когда температура входящего воздуха превышает пороговое значение $(403 \ldots 423) \mathrm{K}$, что было связано с крупномасштабными изменениями фронта пламени. В работе [5] экспериментально исследуется влияние повышенных температур топлива на характеристики горения и выбросы в КС газотурбинного двигателя Rolls Royce $501 \mathrm{~K}$ с модифицированной топливной 
форсункой. Был проведен ряд испытаний с использованием топлива Jet-А при температурах топлива 394 К, 505 К и 590 К.

В экспериментальных исследованиях $[6,7$, 8] показано влияние температуры, давление и состава ГВС на нормальную скорость горения. В [7] результаты измерений сравниваются с результатами численного моделирования. В работах $[9,10]$ приводятся результаты экспериментальных исследований по влиянию температуры входа дизельных топлив на выбросы и характеристики пламени. Имеются также результаты экспериментальных исследований выбросов $\mathrm{NO}_{x}$ при сжигании пылевидных твердых топлив с предварительным подогревом [11].

Среди экспериментальных исследований по воспламеняемости ГВС следует отметить работы $[12,13,14,15,16]$. Эксперименты показали, что диапазон воспламеняемости расширяется с увеличением начальных температур и давления. Верхний предел зависит от давления по логарифмическому закону, а нижний предел воспламеняемости от давления не зависит. От начальной температуры верхний и нижний пределы воспламеняемости зависят линейно [17, 18, 19, 20, 21].

В работах $[22,23]$ содержатся данные по влиянию подогрева топливного газа на показатели эффективности ГТУ.
Информационно-аналитический обзор работ показал, что на сегодняшний день имеются экспериментально-теоретические исследования по влиянию давления и температуры подачи компонентов на концентрационные пределы и на нормальную скорость горения. Особое внимание заслуживает работа [23], в которой расчетным способом показано слабое влияние температуры топлива на режимные параметры ГТУ.

Отличительной особенностью данной работы является, то, что экспериментальным способом показано влияние подогрева топливного газа на эмиссию вредных веществ из камеры сгорания ГТУ. Новизна работы заключается в том, что по результатам расчетно-экспериментальных исследований предлагается новый способ повышения полноты сгорания топлива без изменения газодинамических и геометрических характеристик КС, только подогревом топливного газа.

\section{ОПИСАНИЕ ЭКСПЕРИМЕНТАЛЬНОЙ УСТАНОВКИ}

На рисунке 1 представлена схема экспериментального стенда по исследованию характеристик горения.

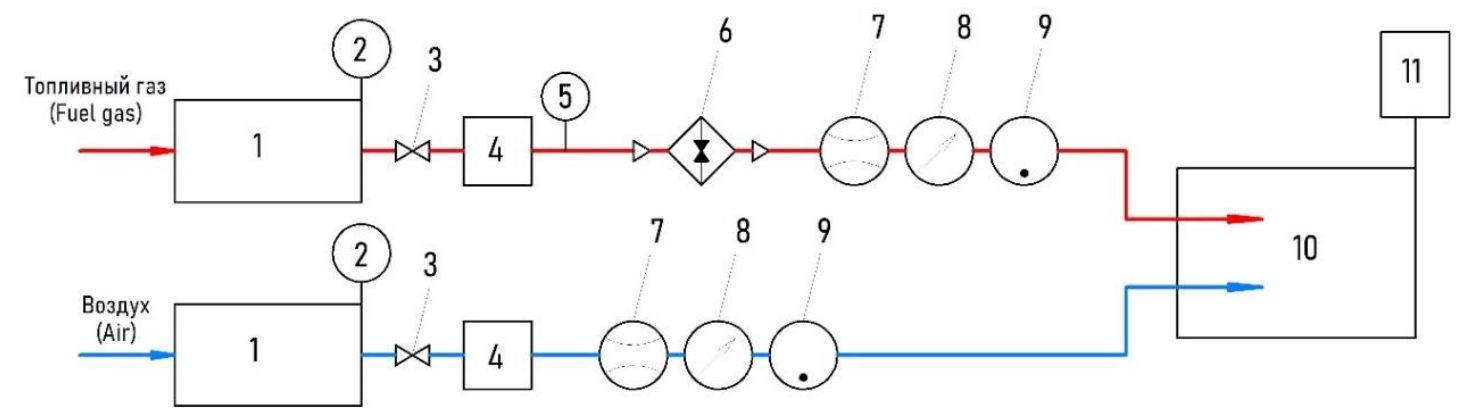

1 - баллон для топливного газа; воздушный ресивер в составе электрокомпрессора; 2 - манометр (давление на входе в редуктор); 3 - вентиль; 4 -редуктор газовый; 5 -манометр (давление на выходе из редуктора); 6- подогреватель топливного газа; 7 - расходомер; 8 - датчик давления; 9 термодатчик; 10 -испытательный отсек с горелочным устройством; 11 - газоанализатор.

1 - fuel gas tank; electrocompressor air receiver; 2 - manometer (pressure at the reducer input); 3 - screw valve; 4 -gas reducer; 5 -manometer (pressure at the reducer output); 6 -fuel gas heater; 7 -flow meter device; 8 -pressure sensor; 9 -thermal sensor; 10 -torch test compartment; 11 - gas analyser.

\section{Рис. 1. Схема экспериментального стенда.}

Fig. 1. Scheme of test bench.

В экспериментальной установке используется редукторная схема подачи воздуха и топливного газа. Топливный газ из баллона 1 через редуктор 4 поступает в электроподогре- ватель 6, где нагревается до заданной температуры подачи в КС. Параметры подогретого топливного газа после подогревателя 6 (объемный расход $Q_{\text {гор }}$, давление подачи $P_{\text {гор }}$ и 
температура подачи $\left.T_{\text {гор }}\right)$ и воздуха после редуктора 4 (объемный расход $Q_{o}$, давление подачи $P_{o \kappa}$ и температура подачи $T_{\text {ок }}$ ) регистрируются расходомерами 7, датчиками давления 8 и термодатчиками 9 и записываются с помощью измерительного комплекса «МЕРА».

На рисунке 2 представлена фотография испытательного отсека, предназначенного для исследования процесса сжигания газообраз- ного топлива, включая природный газ и низкокалорийные газы, такие как синтез-газы, попутные нефтяные газы, доменные газы и т.д. Основной частью испытательного отсека является горелочное устройство (ГУ). ГУ имеет два канала подвода горючего, для организации контуров диффузионного и гомогенного горения.

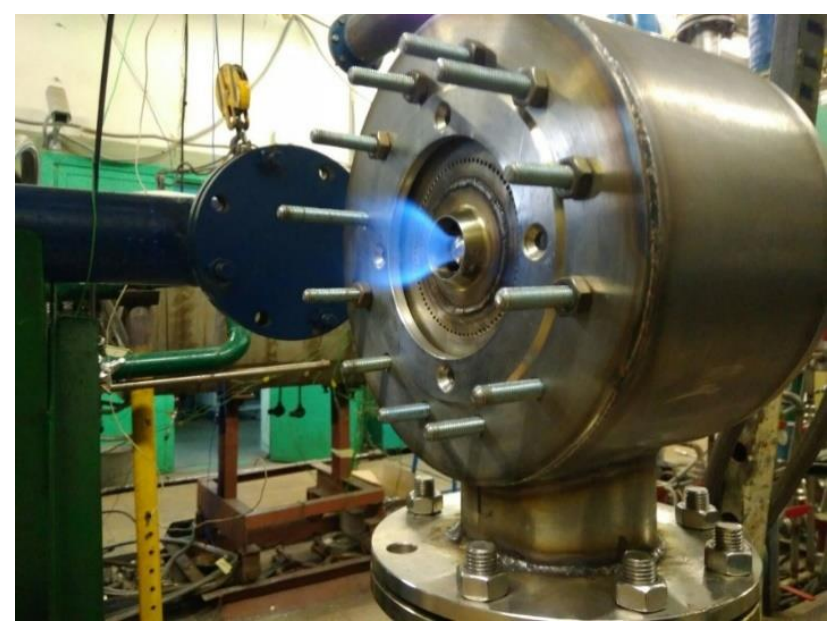

Рис. 2. Испытательный отсек.

Fig. 2. Test compartment.

Измерения эмиссионных характеристик проводились газоанализатором 11 Testo-350. Отбор проб выхлопных газов на анализ осуществлялся через специальный пробоотборник, расположенный в выхлопной магистрали стенда. Температура топливного газа измерялась хромель - копелевой термопарой, установленной на входе в диффузионный канал ГУ.

Для минимизации тепловых перетечек между топливным газом и воздухом на входе в ГУ трубки подвода топливного газа на большей длине были помещены в специальный теплоизолирующий стакан. Однако, как будет показано ниже, полностью исключить их не удалось.

В данной серии экспериментов использовался контур диффузионного горения.

Коэффициент избытка воздуха в условиях эксперимента определялся по параметрам подачи воздуха и топливного газа.

Массовые расходы окислителя и горючего равны:

$$
\begin{gathered}
\dot{m}_{o \kappa}=\rho_{o \kappa} \cdot Q_{o \kappa} ; \\
\dot{m}_{\text {гор }}=\rho_{\text {гор }} \cdot Q_{\text {гоp }},
\end{gathered}
$$

где $Q_{o \kappa}, Q_{\text {гор }}$ - экспериментальные значения объемных расходов.

Плотности воздуха и топливного газа определялись с использованием экспериментальных значений параметров подачи:

$$
\begin{gathered}
\rho_{о \kappa}=\frac{P_{о \kappa}}{R_{o \kappa} \cdot T_{o \kappa}} ; \\
\rho_{\text {гор }}=\frac{P_{\text {гор }}}{R_{\text {гор }} \cdot T_{\text {гор }}},
\end{gathered}
$$

где $P_{\text {ок }}, P_{\text {гор }}$ - экспериментальные значения давления; $T_{\text {ок}}, T_{\text {гор }}$ - экспериментальные значения температуры.

Коэффициент избытка воздуха по условиям подачи компонентов:

$$
\alpha=\frac{1}{K_{m 0}} \cdot \frac{\dot{m}_{\text {ок }}}{\dot{m}_{\text {гор }}},
$$

где $K_{m 0}-$ массовое стехиометрическое соотношение компонентов. 


\section{МЕТОДИКА ИСПЫТАНИЙ И РЕЗУЛЬТАТЫ}

Методикой проведения испытания предусмотрено простое непосредственное сравнение качества горения по уровню эмиссии СО и NOx без подогрева топлива и с его подогревом до максимально возможной величины, исходя из возможностей стендового подогревателя.

Экспериментальные исследования показали, что температура топливного газа на выходе из отверстий форсунок, на входе в зону горения, $T_{3.2}^{6 x}$ выше температуры топливного газа на входе в ГУ $T_{2 y}^{6 x}$ из-за его дополнительного нагрева в каналах корпуса ГУ. Было определено, что на начальном режиме испытаний $T_{z y}^{6 x}=20^{\circ} \mathrm{C} ; T_{3.2}^{B x}=141^{\circ} \mathrm{C}$, а на максимальном режиме испытаний $T_{2 y}^{k x}=120^{\circ} \mathrm{C}$;

$T_{3.2}^{6 x}=169^{\circ} \mathrm{C}$. Указанное обстоятельство привело к нарушению пропорциональности между $T_{3.2}^{6 x}$ и $T_{2 y}^{6 x}$.

В качестве начального (исходного) режима выбран реальный режим работы горелочного устройства в составе камеры сгорания ГТУ, характеризующийся повышенным уровнем эмиссии СО. Параметры исходного режима приведены в таблице 1.

Была проведена серия экспериментальных исследований при осредненных условиях, указанных в таблице 1. Для контроля представительности эксперимента каждая серия исследований завершалась отключением подогрева до восстановления начальной температуры топлива, при которой производились контрольные замеры эмиссии $\mathrm{CO}$ и $\mathrm{NO}_{x}$.

Таблица 1.

Table 1.

Параметры исходного режима работы ГТУ.

Parameters of initial mode of GTU operation.

\begin{tabular}{|c|c|c|c|c|}
\hline № & $\begin{array}{l}\text { Название } \\
\text { (Name) }\end{array}$ & $\begin{array}{l}\text { Обозначение } \\
\text { (Designation) }\end{array}$ & $\begin{array}{l}\text { Размерность } \\
\text { (Dimension) }\end{array}$ & $\begin{array}{c}\text { Значение } \\
\text { (Value) }\end{array}$ \\
\hline 1 & $\begin{array}{c}\text { Абсолютное давление в камере сгорания } \\
\text { (Absolute pressure in the burner) }\end{array}$ & $P_{\kappa}$ & МПа (MPa) & 0.114 \\
\hline 2 & $\begin{array}{l}\text { Температура воздуха на входе в ГУ } \\
\text { (Air temperature at the burner input) }\end{array}$ & $T_{\text {ок }}$ & ${ }^{\circ} \mathrm{C}$ & 257.0 \\
\hline 3 & $\begin{array}{l}\text { Расход воздуха } \\
\text { (Air consumption) }\end{array}$ & $\dot{m}_{o \kappa}$ & кг/c (kg/s) & 0.033 \\
\hline 4 & $\begin{array}{c}\text { Коэффициента избытка воздуха } \\
\text { (Air excess coefficient) }\end{array}$ & $\alpha$ & - & 4.59 \\
\hline 5 & $\begin{array}{c}\text { Температура топливного газа на входе в ГУ } \\
\text { (Fuel gas temperature at the burner input) }\end{array}$ & $T_{z y}^{b x}$ & ${ }^{\circ} \mathrm{C}$ & 20.0 \\
\hline 6 & $\begin{array}{c}\text { Температура топливного газа на выходе из } \\
\text { отверстий форсунки ГУ } \\
\text { (Fuel gat temperature at the output of the burner's } \\
\text { injector holes) }\end{array}$ & $T_{3.2}^{B x}$ & ${ }^{\circ} \mathrm{C}$ & 141.0 \\
\hline 7 & Эмиссия СО (CO emission) & $C_{C O}$ & $\mathrm{M \Gamma} / \mathrm{M}^{3}\left(\mathrm{mg} / \mathrm{m}^{3}\right)$ & 869.0 \\
\hline 8 & Эмиссия $\mathrm{NO}_{\mathrm{x}}\left(\mathrm{NO}_{\mathrm{x}}\right.$ emission $)$ & $C_{N O_{x}}$ & $\mathrm{M \Gamma} / \mathrm{M}^{3}\left(\mathrm{mg} / \mathrm{m}^{3}\right)$ & 77.3 \\
\hline
\end{tabular}

Осредненные результаты испытаний приведены в таблице 2. Величина подогрева топливного газа рассчитывалась по формуле:

$$
\Delta T_{\text {гоp }}=\left(T_{3.2}^{6 x}\right)_{i}-\left(T_{3.2}^{6 x}\right)_{1},
$$

где $\mathrm{i}$ - порядковый номер эксперимента.
Степени изменения эмиссии оксида углерода и окислов азота определялись по формулам:

$$
\begin{aligned}
\Delta C_{C O} & =\left(\frac{C_{C O 1}}{C_{C O i}}-1\right) \cdot 100 \% \\
\Delta C_{N O_{x}} & =\left(1-\frac{C_{N O x 1}}{C_{N O x i}}\right) \cdot 100 \% .
\end{aligned}
$$


На рисунках 4 и 5 показаны графические зависимости осредненных результатов испытаний от величины подогрева топливного газа в ГУ.

Таблица 2.

Table 2.

Осредненные результаты испытаний.

Averaged test results.

\begin{tabular}{|c|c|c|c|c|}
\hline $\mathrm{i}$ & $\begin{array}{c}\text { Величина подогрева } \\
\text { топливного газа } \\
\Delta T_{\text {гор }},{ }^{\circ} \mathrm{C} \\
\text { (Value of fuel gas heat- } \\
\text { up) }\end{array}$ & $\begin{array}{c}\text { Относительная } \\
\text { величина подогрева } \\
\text { топливного газа, \% } \\
\text { (Relative value of the } \\
\text { fuel gas heat-up) }\end{array}$ & $\begin{array}{c}\text { Степень понижения } \\
\text { эмиссии оксида } \\
\text { углерода } \Delta C_{C O}, \% \\
\text { (Degree of decrease in } \\
\text { carbon oxide emission) }\end{array}$ & $\begin{array}{c}\text { Степень повышения } \\
\text { эмиссии окислов } \\
\text { азота } \Delta C_{N O_{x}}, \% \\
\text { (Degree of increase in } \\
\text { nitrogen oxides } \\
\text { emission) } \\
\end{array}$ \\
\hline 1 & 0 & 0 & 0 & 0 \\
\hline 2 & 3.6 & 2.55 & 7.09 & 3.98 \\
\hline 3 & 7.3 & 5.18 & 13.54 & 6.08 \\
\hline 4 & 15.5 & 10.99 & 20.68 & 9.38 \\
\hline 5 & 19.4 & 13.76 & 24.84 & 11.45 \\
\hline 6 & 23.7 & 16.81 & 27.68 & 12.36 \\
\hline 7 & 28.0 & 19.86 & 30.60 & 13.44 \\
\hline
\end{tabular}

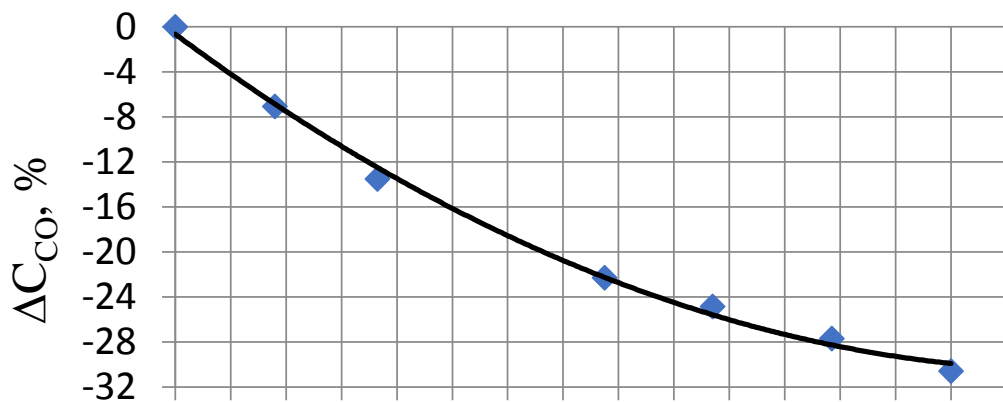

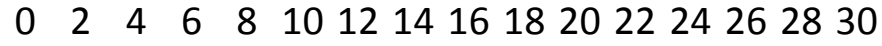

$\Delta \mathrm{T}_{\text {гор }}\left(\Delta \mathrm{T}_{\text {fuel }}\right),{ }^{\circ} \mathrm{C}$

Рис. 3. Осредненная эмпирическая зависимость выбросов СО от величины подогрева топливного газа.

Fig. 3. Averaged empirical dependence of $\mathrm{CO}$ emission on the value of the fuel gas heat-up.

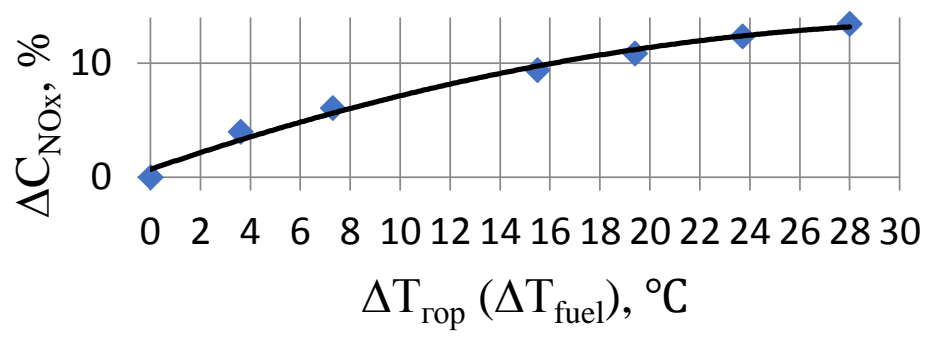

Рис. 4. Осредненная эмпирическая зависимость выбросов NOx от величины подогрева топливного газа.

Fig. 4. Averaged empirical dependence of $\mathrm{NO}_{\mathrm{x}}$ emission on the value of the fuel gas heat-up. 
Как видно из графиков на рисунках 4 и 5 , зависимости выбросов от температуры топлива в исследованном диапазоне температур подогрева топлива близки к линейным. Незначительное отклонение зависимостей от линейных объясняется перетечками тепла между топливом и стенками ГУ из-за недостаточной теплоизоляции топлива в каналах горелочного устройства.

\section{СРАВНИТЕЛЬНЫЙ АНАЛИЗ РЕЗУЛЬТАТОВ ИСПЫТАНИЙ}

Сравнение эффективности альтернативного способа повышения полноты сгорания путем нагрева топлива с традиционными способами - путем повышения температуры воздуха ГВС производилось при условии внесения в ГВС одинакового количества дополнительного тепла и, соответственно, при одинаковых изменениях основных газодинамических параметров, определяющих объем зоны горения, скорость ГВС и время пребывания топлива в зоне горения.

Температура ГВС определялась по уравнению энергетического баланса:

$$
T_{\Gamma B C}=\frac{\alpha \cdot K_{m 0} \cdot T_{o \kappa} \cdot c_{p . o \kappa}+T_{\text {гоp }} \cdot c_{p . z o p}}{\left(1+\alpha \cdot K_{m 0}\right) \cdot c_{p . T B C}} .
$$

Удельные теплоемкости воздуха и топливного газа определялись по аналитическим зависимостям, которые получены методом

наименьших квадратов при анализе табличных данных:

$$
\begin{aligned}
& c_{\text {p.ок }}=0.181 \cdot T_{\text {ок }}+950.6 ; \\
& c_{\text {p.гор }}=3.273 \cdot T_{\text {гор }}+1251 .
\end{aligned}
$$

Удельная теплоемкость ГВС определялась в зависти от соотношения компонентов по формуле:

$$
c_{p . \Gamma B C}=\frac{\alpha \cdot K_{m 0} \cdot c_{p . o \kappa}+c_{p .2 o p}}{1+\alpha \cdot K_{m 0}} .
$$

Миделевая скорость ГВС определялась по формуле [20]:

$$
W_{\Gamma B C}=\frac{\dot{m}_{\Gamma B C}}{\rho_{T B C} \cdot F_{K C}} .
$$

Расходонапряженность определялась по формуле:

$$
g_{k}=\frac{W_{\Gamma B C}}{R_{\Gamma B C} \cdot T_{\Gamma B C}} .
$$

В таблице 3 отражены основные изменения введенные в газодинамику КС при подогреве

\begin{tabular}{|c|c|c|c|c|c|c|}
\hline $\begin{array}{c}\text { № } \\
\Pi / \Pi\end{array}$ & $\begin{array}{c}\text { Величина } \\
\text { подогрева } \\
\text { топливного } \\
\text { газа } \Delta T_{\text {гор }},{ }^{\circ} \mathrm{C} \\
\text { (Value of fuel } \\
\text { gas heat-up) }\end{array}$ & $\begin{array}{c}\text { Величина } \\
\text { подогрева } \\
\text { ГВС } \\
\Delta T_{\Gamma B C},{ }^{\circ} C \\
\text { (Value of } \\
\text { FAM heat- } \\
\text { up) }\end{array}$ & $\begin{array}{c}\text { Скорость } \\
\text { топливного } \\
\text { газа в } \\
\text { отверстиях } \\
\text { форсунки, } \\
\text { м/c } \\
\text { (Rate of fuel } \\
\text { gas in } \\
\text { injector } \\
\text { holes, m/s) }\end{array}$ & $\begin{array}{c}\text { Миделевая } \\
\text { скорость } \\
\text { ГВС, м/c } \\
\text { (Midship } \\
\text { FAM rate, } \\
\text { m/s) }\end{array}$ & $\begin{array}{c}\text { Расходо- } \\
\text { напряженность, } \\
\text { кг / (c·H) } \\
(\text { Flow rate, } \\
\mathrm{kg} /(\mathrm{s} \cdot \mathrm{N}))\end{array}$ & $\begin{array}{c}\text { Время } \\
\text { пребывания } \\
\text { топлива в } \\
\text { камере } \\
\text { сгорания, с } \\
\text { (Staying time } \\
\text { of fuel in the } \\
\text { burner, s) }\end{array}$ \\
\hline 1 & 0 & 0 & 0.127 & 2.667 & $0.175 \cdot 10^{-4}$ & 0.148 \\
\hline 2 & 3.6 & 0.49 & 0.128 & 2.670 & $0.175 \cdot 10^{-4}$ & 0.148 \\
\hline 3 & 7.3 & 1.11 & 0.129 & 2.670 & $0.175 \cdot 10^{-4}$ & 0.148 \\
\hline 4 & 15.5 & 1.78 & 0.131 & 2.671 & $0.175 \cdot 10^{-4}$ & 0.148 \\
\hline 5 & 19.4 & 2.37 & 0.133 & 2.671 & $0.175 \cdot 10^{-4}$ & 0.148 \\
\hline 6 & 23.7 & 3.06 & 0.134 & 2.671 & $0.174 \cdot 10^{-4}$ & 0.148 \\
\hline 7 & 28 & 3.83 & 0.135 & 2.672 & $0.174 \cdot 10^{-4}$ & 0.148 \\
\hline
\end{tabular}
топлива.

Таблица 3.

Table 3.

Основные изменения газодинамических параметров при подогреве топлива.

Main changes in gas-dynamic parameters at fuel heat-up. 
Анализ данных таблицы 3 показывает, что подогрев топливного газа практически не влияет на газодинамические параметры ГВС, что позволяет сделать вывод о неизменности параметров зоны горения, таких как объем зоны горения; доля воздуха, участвующая в горении; время пребывания топлива в зоне горения и др.

Для расчета выбросов оксидов азота $\mathrm{NO}_{x}$ и углерода СО при инженерном моделировании рабочего процесса обычно используют эмпирические зависимости.

В 1981 году Дж. Д. Льюис опубликовал выражение для прогноза $\mathrm{NO}_{x}$ на основе экспериментальных данных о пламени:

$$
C_{N O_{x}}=7.50 \cdot 10^{-6} \cdot e^{\left(8.28 \cdot 10^{-3} \cdot T_{3.2}\right)}, \text { oб.\%, }
$$

где $T_{3.2}$ - температура в зоне горения, К.

Это выражение является функцией только температуры пламени. Позднее Дж. Д. Льюис опубликовал полуэмпирическую формулу [24], которая кроме температуры пламени учитывает давление в камере сгорания и используется при бедном гомогенном горении:

$$
C_{N O_{x}}=3.32 \cdot 10^{-6} \cdot e^{\left(0.008 \cdot T_{3.2}\right)} \cdot P^{0,5}, \text { об.\%, }
$$

где $P$ - давление в камере, кгс/ $\mathrm{cm}^{2}$.

Лефевр А.Н. в [25] для расчета эмиссии СО предложил экспериментальную зависимость:

$$
C_{C O}=\frac{86 \cdot \dot{m}_{0 \kappa} \cdot T_{3.2} \cdot e^{-\left(0.00345 \cdot T_{3.2}\right)}}{\left(V_{3.2}-V_{e}\right)\left(\frac{\Delta P}{P}\right)^{0.5} P^{1.5}}, \frac{2}{\kappa 2},
$$

где $\dot{m}_{\text {ок }}-$ массовый расход воздуха, поступающего в $К С ; ~ \Delta P / P$ - относительные потери давления вдоль камеры $V_{3.2}-$ общий объем зоны горения; $V_{e}-$ объем испарившихся капель (для горения газов $\left.V_{e}=0\right)$.

Также Лефевр А.Н. в [26] предложил использовать для расчета эмиссии СО следующую уточненную формулу:
$C_{C O}=\frac{20 \cdot f^{2} \cdot \dot{m}_{\text {ок.3.2. }} \cdot T_{n .2}\left(\frac{\Delta P}{P}\right)^{0.5} \cdot P}{V_{3.2} \cdot e^{\left(0.009 \cdot T_{3.2}\right)}} \cdot 10^{-4}$, об.\%,

где $f$ - доля воздуха, участвующая в горении; $\dot{m}_{\text {ок.з.2 }}-$ расход воздуха в зоне горения; $T_{n .2}-$ температура продуктов сгорания на выходе из камеры.

Ризк Н.К. в [27] для расчета эмиссии СО использовал следующую зависимость:

$$
C_{C O}=\frac{0.18 \cdot 10^{9} \cdot e^{\left(\frac{7800}{T_{3.2}}\right)}}{P^{2} \cdot\left(\tau-0.4 \cdot \tau_{e}\right)\left(\frac{\Delta P}{P}\right)^{0.5}}, \frac{2}{\kappa 2},
$$

где $\tau$ - время пребывания в зоне горения; $\tau_{e}-$ время испарения капли горючего.

Сделанный ранее вывод о неизменности параметров зоны горения от величины подогрева топливного газа, дает право воспользоваться указанными выше эмпирическими зависимостями для оценки влияния подогрева топливного газа на полноту сгорания топлива в относительных единицах. При этом все члены, не зависящие от $T_{3.2}$ обозначены в виде коэффициентов $K_{1}, K_{2}, K_{3}$. Тогда уравнения (1), (2), (3) соответственно примут следующий вид:

$$
\begin{gathered}
C_{N O_{x}}=K_{1} \cdot e^{\left(0.008 \cdot T_{3.2}\right)} ; \\
C_{C O}=K_{2} \frac{1}{e^{\left(0.009 \cdot T_{3.2}\right)}} ; \\
C_{C O}=K_{3} \cdot e^{\left(\frac{7800}{T_{3.2}}\right)} .
\end{gathered}
$$

На рисунках 6 и 7 представлены зависимости изменения концентраций $\mathrm{CO}$ и $\mathrm{NO}_{\mathrm{x}}$ от величины подогрева ГВС, рассчитанные по выражениям (4), (5), (6) соответственно.

Сравнение данных по эмиссии $\mathrm{CO}$ на рисунках 4 и 7 и данных по эмиссии $\mathrm{NO}_{x}$ на рисунках 5 и 6 показывает, что подогрев топливного газа значительно эффективнее подогрева воздуха по влиянию на полноту сгорания топлива. 


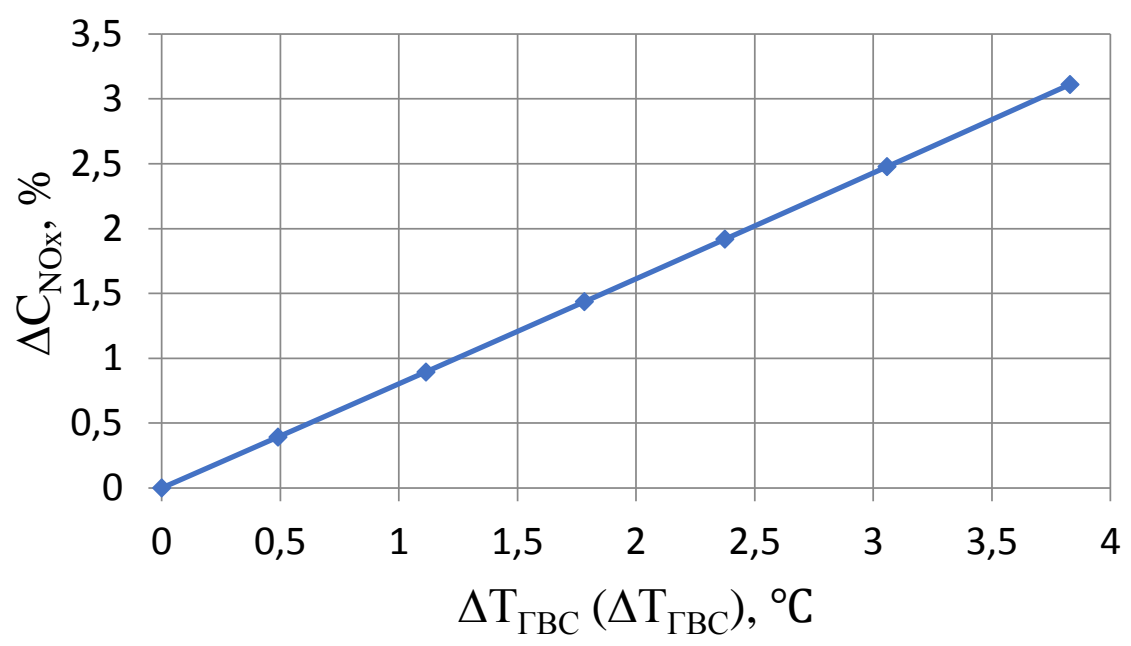

Рис. 5. Зависимость изменения концентрации NOх от температуры TBC.

Fig. 5. Dependence of $\mathrm{NO}_{x}$ concentration change on FAM temperature.

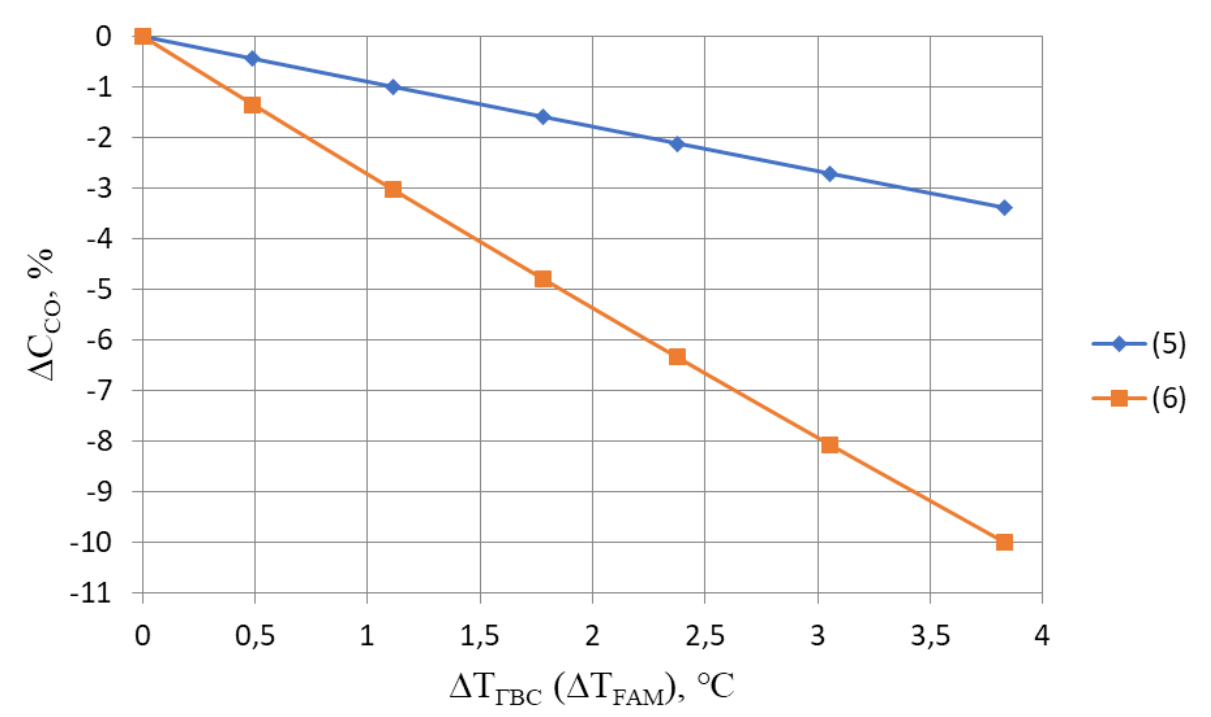

Рис. 6. Зависимость изменения концентрации СО от температуры ТВС.

Fig. 6. Dependence of $\mathrm{CO}$ concentration change on FAM temperature.

\section{ЗАКЛЮЧЕНИЕ}

Проведенные расчетно-экспериментальные исследования по влиянию подогрева топливного газа на газодинамические и эмиссионные характеристики горения позволяют сформулировать следующие выводы:

- внешний подогрев топливного газа незначительно влияет на термо- и газодинамические характеристики горения. Получено, что повышение температуры горюче-воздушной смеси и продуктов сгорания составляет примерно 0.15 гардуса на 1 градус подогрева топливного газа;

- показана сильная зависимость эмиссии $\mathrm{CO}$ и $\mathrm{NO}_{\mathrm{x}}$ от внешнего подогрева топливного газа. Получено, что уменьшение эмиссии СО составляет $1.1 \%$, а увеличение эмиссии $\mathrm{NO}_{x}$ $0.5 \%$ на 1 градус подогрева топливного газа;

- полученные результаты свидетельствуют о возможности реализации нового способа регулирования газотурбинных установок путем подогрева топливного газа с регистрацией выбросов $\mathrm{NO}_{x}$ и $\mathrm{CO}$ без изменения расходных и конструктивных характеристик камеры сгорания.

\section{Литература (References)}

[1] Ueli Honegger. Gas turbine combustion modeling for a parametric emissions monitoring system. Thesis, 2007

[2] Pinaki Pal. Computational modeling and analysis of low temperature combustion regimes for advanced engine applications. Thesis, 2016 
[3] Wang, F.-S \& Kong, Wenjun \& Wang, B.-R \& Lao, S.-Q \& Zhang, P.-Y. (2007). Effects of inlet temperature on combustion characteristics within a micro gas turbine combustor. Kung Cheng Je $\mathrm{Wu} \mathrm{Li}$ Hsueh Pao/Journal of Engineering Thermophysics. 28. 331-334.

[4] Aerospace Science and Technology, Volume 92, September 2019, Pages 126-138, Aerospace Science and Technology Effects of fuel variation and inlet air temperature on combustion stability in a gas turbine model combustor Feier Chen Can Ruan Tao Yu Weiwei Cai Yebing Mao Xingcai Lu https://doi.org/10.1016/j.ast.2019.05.052

[5] Wiest, H.K. \& Heister, S.D.. (2014). Experimental study of gas turbine combustion with elevated fuel temperatures. Journal of Engineering for Gas Turbines and Power. 136. 10.1115/1.4027907

[6] Zhao Z., Kazakov A., Li J., Dryer F.L. The initial temperature and $\mathrm{N}-2$ dilution effect on the laminar flame speed of propane/air. Combustion Science and Technology, 2004, vol. 176(10), pp. 1705-23, doi: 10.1080/00102200490487553

[7] Natarajan J., Kochar Y., Lieuwen T., Seitzman J. Pressure and preheat dependence of laminar flame speeds of $\mathrm{H} 2 / \mathrm{CO} / \mathrm{CO} 2 / \mathrm{O} 2 / \mathrm{He}$ mixtures. Proceedings of the Combustion Institute, 2009, vol. 32(1), pp. 1261-1268, doi: 10.1016/j.proci.2008.06.110

[8] Combustion and Flame, Volume 158, Issue 8, August 2011, Pages 1491-1506 Homogeneous combustion of fuel-lean $\mathrm{H} 2 / \mathrm{O} 2 / \mathrm{N} 2$ mixtures over platinum at elevated pressures and preheats Yohannes Ghermaya John Mantzarasa Rolf Bombacha Konstantinos Boulouchosb https://doi.org/10.1016/j.combustflame.2010.12.0 25

[9] Chen, Hao \& Guo, Qi \& Zhao, Xu-yi \& Xu, Meng-long \& Ma, Yong. (2015). Influence of Fuel Temperature on Combustion and Emission of Biodiesel. Journal of the Energy Institute. 89. 10.1016/j.joei.2015.01.024

[10] Poorhoseini, Hadi \& Nikzad, R.. (2019). An experimental study on the effect of inlet diesel fuel temperature on mass flow choking, flame penetration, temperature, radiative characteristics and NOx pollutant emission of an oil burner. Journal of the Brazilian Society of Mechanical Sciences and Engineering. 41. 10.1007/s40430019-2022-2

[11]Fuel, Volume 294, 15 June 2021, 120538, Experimental study on NOx emissions of pulverized coal combustion preheated by a $2 \mathrm{MW}$ novel self-sustained preheating combustor Ziqu Ouyangab Wenhao Songab Jingzhang Liua Jianguo Zhuab Chengbo Mana Shujun Zhuac Hongliang https://doi.org/10.1016/j.fuel.2021.120538
[12]Liao S.Y., Cheng Q., Jiang D.M., Gao J. Experimental study of flammability limits of natural gas-air mixture. Journal of Hazardous Materials, 2005, vol. 119, Issues 1-3, pp. 81-84

[13] Gan C., Zili L., Chao Y. Experimental study of flammability limits of methane/air mixtures at low temperatures and elevated pressures. Fuel, 2016, vol. 181, pp. 1074-1080

[14] Wang T., Luo Z., Wen H., Cheng F., Deng J., Zhao J., Guo Z., Lin J., Kang K., Wang W. Effects of flammable gases on the explosion characteristics of $\mathrm{CH} 4$ in air. Journal of Loss Prevention in the Process Industries, 2017, vol. 49, Part B, pp. 183-190

[15]Zamashchikov V.V. On the Flammability Limit. Combustion Explosion and Shock Waves, 2018, vol. 54, Issue 4, pp. 393-397

[16] Catoire L., Naudet V. Estimation of temperature dependent lower flammability limit of pure organic compounds in air at atmospheric pressure. Process Safety Progress, 2005, vol. 24 (2), pp. $130-137$

[17] Wierzba I., Kilchyk V. Flammability limits of gydrogen-carbon monoxide mixtures at moderately elevated temperatures. International Journal of Hydrogen Energy, June 2001, vol. 26, Issue 6, pp. 639-643

[18] Gibbon H.J., Wainwright J., Rogers R.L. Experimental determination of flammability limits of solvents at elevated temperatures and pressures. In Institution of Chemical Engineers Symposium Series, 1994, vol. 134, pp. 1-12

[19]Li Z., Gong M., Sun E., Wu J., Zhou Y. Effect of low temperature on the flammability limits of methane/nitrogen mixtures. Energy, 2011, vol. 36(9), Yuan, pp. 5521-5524.

[20] Ale B.B., Wierzba I. The flammability limits of hydrogen and methane in air at moderately elevated temperatures. Published in: IECEC-97 Proceedings of the Thirty-Second Intersociety Energy Conversion Engineering Conference (Cat. No. 97CH6203)

[21] Bolshova T.A., Bunev V.A., Knyazkov D.A., Korobeinichev O.P., Chernov A.A., Shmakov A.G., Yakimov S.A. Dependence of the lower flammability limit on the initial temperature. Combustion, Explosion, and Shock Waves, March 2012, vol. 48, Issue 2, pp. 125-129

[22] Mehaboob Basha, Shaahid S.M., Luai AlHadhrami. Impact of Fuels on Performance and Efficiency of Gas Turbine Power Plants. 2nd International Conference on Advances in Energy Engineering December 27-28, 2011, Bangkok, Thailand. Energy Procedia 14 (2012),pp. 558-565.

[23] Marin G., Mendeleev D., Osipov B., Akhmetshin A. Study of the effect of fuel temperature on gas turbine performance. Web of Conferences 178, 01033 (2020). 
[24] Lewis, G. D., 1981, "Prediction of NOx Emissions," American Society of Mechanical Engineers (Paper), (81) pp. 5.

[25] Lewis, G. D., 1991, "New Understanding of NOx Formation," Tenth InternationalSymposium on Air Breathing Engines, Anonymous Publ by AIAA, Washington, DC,USA, Nottingham, Engl, pp. 625-629.

[26] Lefebvre, A. H., 1984, "Fuel Effects on Gas Turbine Combustion - Liner Temperature,Pattern Factor and Pollutant Emissions."

\section{Сведения об авторах.}
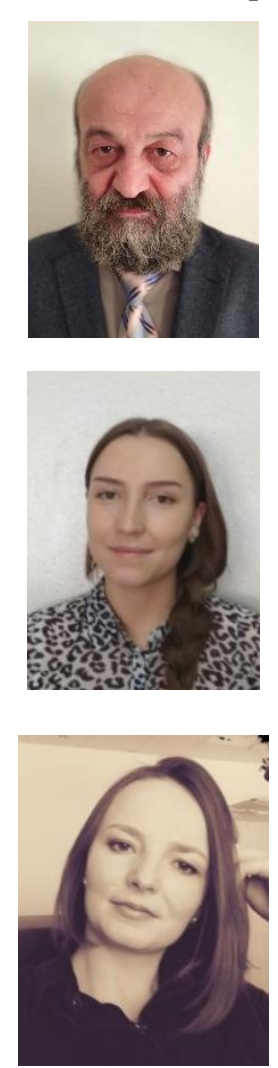

Пеков Ахиллей Периклович - советник генерального директора ПАО НТЦ "Турбопневматик". Область научных интересов: теоретические и экспериментальные исследования внутрикамерных процессов.

E-mail: Pekov3@yandex.ru

Шилова Алена Алексеевна инженер кафедры «Ракетнокосмическая техника и энергетические системы» ПНИПУ. Область научных интересов: рабочие процессы в КС ЭУ. E-mail: alyona1203@gmail.com

\section{Бетинская Оксана Андре-} евна - к.т.н, доцент кафедры «Ракетно-космическая техника и энергетические системы» ПНИПУ. Область научных интересов: турбомашины и комбинированные ЭУ.

E-mail: oksa-
AIAA/SAE/ASME 20th Joint PropulsionConference. Journal of Aircraft, ed. AIAA, New York, NY, USA, Cincinnati, OH, Engl, pp. 18.

[27]Rizk, N. K., and Mongia, H. C., 1993, "Semianalytical Correlations for $\mathrm{NOx}, \mathrm{CO}$, and UHC Emissions," Journal of Engineering for Gas Turbines and Power, Transactions of the ASME, 115(3) pp. 612-619.

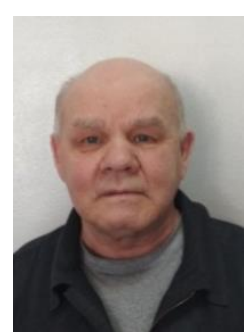

Бачев Николай Леонидович к.т.н., доцент кафедры «Ракетнокосмическая техника и энергетические системы» ПНИПУ. Область научных интересов: утилизационные газотурбинные установки.

E-mail: bn154@yandex.ru

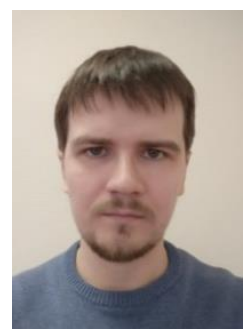

Матюнин Олег Олегович - старший преподаватель кафедры «Ракетно-космическая техника и энергетические системы» ПНИПУ. Область научных интересов: численное моделирование рабочих процессов в ЭУ.

E-mail: matoleg@gmail.com 